\title{
Edible vegetables as a source of aldose reductase differential inhibitors
}

Francesco Balestri ${ }^{1}$, Carlo Sorce ${ }^{1,2}$, Roberta Moschini ${ }^{1,2}$, Mario Cappiello ${ }^{1,2}$, Livia Misuri ${ }^{1,3}$ Antonella Del Corso ${ }^{1,2}$ and Umberto Mura*1,2.

${ }^{1}$ University of Pisa, Department of Biology, Pisa, Italy;

${ }^{2}$ Interdepartmental Research Center Nutrafood “Nutraceuticals and Food for Health”, University of Pisa, Pisa, Italy

${ }^{3} \mathrm{PhD}$ student of the Tuscany Region PhD School in Biochemistry and Molecular Biology *Corresponding author: Umberto Mura, Department of Biology, Biochemistry Unit, Via S. Zeno,51, 56126, Pisa, Italy; Tel: 39. 050.2211451; Fax: 39.050.2211460; E-mail: umberto.mura@unipi.it

\section{Acknowledgments}

This work was supported by Regione Toscana (Italy), Progetto IDARA.

Key words: aldo-keto reductases; aldose reductase; differential inhibitors; Phaseolus vulgaris; functional foods.

\begin{abstract}
Abbreviations
AR, aldose reductase; ARIs, aldose reductase inhibitors; ARDIs, aldose reductase differential inhibitors; HNE, trans-4-hydroxy-2,3-nonenal; DTT, D,L-dithiothreitol; $h \mathrm{AR}$, human placental recombinant aldose reductase; GAL, D,L-glyceraldehyde; EDTA, ethylenediaminetetraacetic disodium salt; BSA, bovine serum albumin.
\end{abstract}

\footnotetext{
Abstract

The hyperactivity of aldose reductase (AR) on glucose in diabetic conditions or on glutathionylhydroxynonenal in oxidative stress conditions, the source of cell damage and inflammation, appear to be balanced by the detoxifying action exerted by the enzyme. This detoxification acts on cytotoxic hydrophobic aldehydes deriving from membrane peroxidative processes. This may contribute to the failure in drug development for humans to favorably intervene in diabetic complications and inflammation, despite the specificity and high efficiency of several available
} 
aldose reductase inhibitors. This paper presents additional features to a previously proposed approach, on inhibiting the enzyme through molecules able to preferentially inhibit the enzyme depending on the substrate the enzyme is working on. These differential inhibitors (ARDIs) should act on glucose reduction catalyzed by AR without little or no effect on the reduction of alkenals or alkanals. The reasons why AR may be an eligible enzyme for differential inhibition are considered. These mainly refer to the evidence that, although AR is an unspecific enzyme that recognizes different substrates such as aldoses and hydrophobic aldehydes, it nevertheless displays a certain degree of specificity among substrates of the same class. After screening on edible vegetables, indications of the presence of molecules potentially acting as ARDIs are reported.

\section{Introduction}

More than 50 years have passed since the hyperosmotic theory of the galactose-induced cataract linked to aldose reductase activity was first proposed [1]. At that time, it really looked like a short cut to finding tools to counteract diabetic complications. In fact, as formulated, the control of polyol formation through AR inhibition, appeared to be a logical intervention in secondary complications of diabetes. Besides the osmotic stress, other damaging events may be associated with an enhanced flux of the polyol pathway. These include a substantial alteration of the physiological ratios of the redox couples of both NAD and NADP, with a consequent decrease in antioxidant defense ability [2] and, finally, a potential increase in protein glycation phenomena due to an increase in fructose levels [3-5].

It thus appeared reasonable that AR needed to be inhibited and great experimental efforts began which are still on-going [6]. Indeed, hundreds of ARIs have been characterized but no significant drug development has followed. The case of Sorbinil, which did not pass clinical trials is emblematic [7] as is the withdrawal of Tolrestat-based drugs from distribution [8]. Today, to our knowledge, except for some eastern countries where Epalrestat-based drugs have been commercialized, no ARI-based drugs are used for human care in the West.

There may be several reasons for such a failure, from the lack of bioavailability of the drug to the severe side effects, however they may also be somehow related to the catalytic features of the enzyme itself, as AR can reduce toxic aldehydes derived from lipid peroxidative processes $[9,10]$. Thus, unlike the potential damage of increasing the polyol pathway flux, the removal of 4-hydroxy2-nonenal (HNE), a significant product in membrane peroxidative processes, and other alkanals and alkenals, must be considered as a detoxifying function of the enzyme [11]. In addition, the ability of the AR to reduce glutathionyl-hydroxynonenal (GSHNE), the most important HNE derivative, 
highlights a link between AR activity and the pro-inflammatory cell response $[10,12,13]$. It is therefore not easy to predict the overall effect of the enzyme inhibition.

A change in the approach to the enzyme inhibition has recently been proposed, that has moved away to a certain extent from the search for extreme inhibitory power in ARIs, but aimed at specifically intervening in the reaction that the enzyme is catalyzing in a differential manner [14].

Consequently, differential inhibitors, ARDIs, as these molecules were called, should intervene in glucose and or GSHNE reduction, with no or a reduced effect on HNE and other alkanal/alkenal reduction.

The potential of inhibitors to act as ARDIs is linked to their inhibition model, as the targeting of the free enzyme (i.e. a competitive type of inhibition) is an important feature of these molecules. While a mixed type of differential inhibition, especially when the targeting on the free enzyme is the process preferred, is still able to differentially affect the transformation of the two agonist substrates, uncompetitive differential inhibitors cannot exert any differential inhibitory action [15]. This paper, revisiting previous observations on AR substrate specificity features, deals with the rational explanation as to why AR is an eligible target for differential inhibition. Preliminary, though important indications of edible vegetables as potential ARDIs sources are reported.

\section{2-Materials and Methods}

2.1 Materials. Bovine serum albumin (BSA), D,L-dithiothreitol (DTT),D,L-glyceraldehyde (GAL), EDTA, were purchased from Sigma-Aldrich (Saint Louis, MO, USA). NADPH, L-idose, D-glucose and L-glucose were supplied by Carbosynth (Compton, England); YM10 ultrafiltration membranes were obtained from Merck-Millipore (Darmstad, Germany); HNE was prepared as previously described [16]. All other chemicals were of reagent grade. Dry beans of the yellow Zolfino landrace were obtained from "Agostinelli Mario" farm in Leccio-Reggello (Florence, Italy); "Costoluto fiorentino" tomato (Solanum lycopersicum) and "Black" cabbage (Brassica oleracea var. sabellica) were obtained from the Le Prata farm in S.Giuliano (Pisa, Italy).

\subsection{Assay of aldose reductase.}

The activity of AR was determined at $37^{\circ} \mathrm{C}$ as previously described [17], following the decrease in absorbance at $340 \mathrm{~nm}$ due to NADPH oxidation $\left(\varepsilon_{340}=6.22 \mathrm{mM}^{-1} \mathrm{~cm}^{-1}\right)$. The standard assay mixture contained a $0.25 \mathrm{M}$ sodium phosphate buffer $\mathrm{pH}$ 6.8, $0.18 \mathrm{mM}$ NADPH, 2.4 M ammonium sulfate, $0.5 \mathrm{mM}$ EDTA and 4.7 mM GAL. One unit of enzyme activity is the amount that catalyzes the conversion of $1 \mu \mathrm{mol}$ of substrate/min in the above assay conditions. These assay conditions were 
also adopted to evaluate the effectiveness as inhibitors of the vegetable extracts when either $0.6 \mathrm{mM}$ L-idose, or $0.04 \mathrm{mM}$ HNE, were used as a substrate instead of GAL.

\subsection{Purification of human recombinant AR.}

The $h \mathrm{AR}$ was expressed and purified as previously described [18]. The purity of the final enzyme preparation was assessed by SDS-PAGE [19] and gels were stained with silver nitrate [20], which showed a unique band corresponding to a molecular weight of approximately $34 \mathrm{KDa}$. The specific activity of purified $h \mathrm{AR}$ was $5.3 \mathrm{U} / \mathrm{mg}$. The purified enzyme was stored at $-80{ }^{\circ} \mathrm{C}$ in a $10 \mathrm{mM}$ sodium phosphate buffer $\mathrm{pH} 7.0$ containing $2 \mathrm{mM}$ DTT and $30 \%(\mathrm{w} / \mathrm{v})$ glycerol. Before use, the enzyme was extensively dialyzed against the $10 \mathrm{mM}$ sodium phosphate buffer $\mathrm{pH}$ 7.0.

\subsection{Vegetable extract preparation}

Fresh vegetables and dried beans were mechanically disrupted by Ultra-Turrax and extracted by $80 \%$ methanol aqueous solution containing $0.6 \%(\mathrm{w} / \mathrm{v})$ of acetic acid. The extraction was performed by the addition of $5 \mathrm{ml}$ of the above methanol solution per $\mathrm{g}$ of the sample. The suspension was kept for approximately $5 \mathrm{~h}$ at room temperature, centrifuged for $10 \mathrm{~min}$ at $4{ }^{\circ} \mathrm{C}$ at $7,000 \mathrm{xg}$, and the pellet extracted again at $4{ }^{\circ} \mathrm{C}$ overnight as above. The supernatants of the two centrifugation steps were pooled and dried at room temperature by a rotary evaporator. The dried samples were suspended in a $10 \%$ methanol aqueous solution containing $0.6 \%(\mathrm{w} / \mathrm{v})$ of acetic acid $(2 \mathrm{ml} / \mathrm{g}$ equivalents of the original sample weight) and filtered through $0.45 \mu \mathrm{m}$ PTFE membrane filters. An aliquot $(0.5 \mathrm{ml})$ was then loaded on a reverse phase HPLC column (Phenomenex Kinetex C18, 250 $\mathrm{x} 4.6 \mathrm{~mm} \mathrm{ID,} 5 \mu \mathrm{m}$ particle size). Elution was performed at a constant flux of $1 \mathrm{ml} \mathrm{min}^{-1}$ for $4 \mathrm{~min}$ before applying a linear gradient, from 10 to $100 \%$ in $23 \mathrm{~min}$, of methanol containing $0.6 \%$ acetic acid. Fractions of $2 \mathrm{ml}$ were collected. Elution was monitored by an online detector set at $254 \mathrm{~nm}$. Corresponding fractions from different runs were pooled, dried by a speedvac apparatus, and suspended in water in order to perform AR inhibition assays.

\subsection{Other methods.}

The protein concentration was determined according to Bradford [21], using BSA as a standard protein.

Statistical analysis was performed using the two-way ANOVA test operated with Graphpad 6.0 software. 


\section{Results and Discussion}

\section{1 - AR as a target of differential inhibition.}

The possible intra-site differential inhibition of enzymes poses the question as to what the essential requirement is for the target enzyme to be susceptible to this kind of inhibitory action. It is clear that the differential inhibition strategy to control enzyme activity may take place only for enzymes that are able to act on different substrates. However, this is not sufficient to make the enzyme eligible for differential inhibition targeting, since the enzyme may specifically recognize a very limited portion of different substrate molecules, which is likely to be the one undergoing transformation. Thus the lack of substrate specificity for an enzyme is a necessary but not sufficient condition to make it susceptible to differential inhibition.

A strict requirement for an enzyme to be targeted through differential inhibition is its ability to make different interaction pathways available for two agonist substrates, one of which can thus be exclusively, or at least differently, interdicted by the inhibitor with respect to the other one. It is known that AR is able to act on structurally very different substrates, such as aldoses and alkanals/alkenals. However, despite apparently possessing the features of an aldehyde removase, $\mathrm{AR}$ is not a permissive enzyme. In fact it is able to display a different degree of catalytic efficiency toward substrates of the same class $[22,23]$. Clear evidence of this is shown in Table 1, where the relative specificity constants for members of two different classes of AR substrates (i.e. highly hydrophilic aldoses and hydrophobic alkenals and alkanals) are reported.

While the specificity constants for hydrophobic substrates are relative to the least efficient one, in order to avoid the effect due to possible differences in the free aldehyde form of different aldoses, the values for hydrophilic aldoses are reported as the $k_{s}$ ratio of the two D and L enantiomers. For both classes of substrates, a significant difference was observed in the efficiency of the enzyme to catalyze their reduction, up to approximately 2.5 orders of magnitude for alkanals and alkenals, and approximately 50 fold for aldose enantiomers. The case of the D- and L- enantiomeric couples for glucose and idose is emblematic as highlighted in Fig. 1. In fact, D-glucose and its epimer at C5, Lidose, are substrates for AR with a specificity constant of 0.61 and $35.75 \mathrm{mM}^{-1} \mathrm{~min}^{1}$, respectively [18]. On the other hand, L-glucose and its epimer at C5, D-idose, are both poor substrates for the enzyme. The poor effectiveness of L-glucose as substrate [24] makes it hard to kinetically characterize this substrate, making the relative specificity constant for the L/D glucose couple difficult to be evaluated. On the contrary for D-idose, a $k_{c a t}$ of $145.56 \pm 13.16 \mathrm{~min}^{-1}$ and a $K_{M}$ of $34.45 \pm 5.83 \mathrm{mM}$ were measured, with a resulting specificity constant approximately one order of magnitude lower with respect to L-idose (Table 1). 
These data suggest that a special recognition pattern must occur both for aldoses and for hydrophobic substrates; these patterns should be specific for each substrate typology and it may be possible to envisage specific inhibitors. Another particular aspect that distinguishes the targeting of the enzyme while catalyzing the aldose reduction with respect to the hydrophobic aldehyde reduction, is the non-hyperbolic inhibition exerted on the enzyme by haemiacetals [25]. In this regard it would be advisable to search for differential inhibitors in conditions in which both agonist substrates were present. Although it might be a less friendly assay condition with respect to the direct monitoring of the absorbance at $340 \mathrm{~nm}$, the double substrate assay approach, while truly pairing the assay conditions for both substrates would reveal possible favorable evidence in the search for ARDIs. In fact the differential targeting of one of the two reactions elicits the other reaction, because of the removal, or of the attenuation, of the reciprocal competitive inhibition that the two agonist substrates exert on each other.

\section{2 - Differential inhibitors in edible vegetables. Although evidence of the susceptibility of AR to} differential inhibition has been presented using synthetic molecules [14], the search for ARDIs is not easy, due to the intrinsic difficulty in finding ARIs with the additional restrictive features required for a differential intra-site inhibition. Generally the development of new chemical entities into drugs for human use is a very long and expensive process. Natural products, however, remain a valuable alternative in developing drugs, or in generating commercial preparations enriched with natural healthy molecules or at least in promoting the use of functional food for human health. Here a first approach in searching for ARDIs in plants is presented.

Three different edible vegetable species, namely, "Zolfino" bean, a variety of Phaseolus vulgaris L. from Pratomagno, "Costoluto fiorentino" tomato (Solanum lycopersicum) and "Black" cabbage (Brassica oleracea var. sabellica), typical of the Tuscany region (Italy), were used to evaluate the possible occurrence of ARDIs. The susceptibility to inhibition of AR while catalyzing the reduction of either L-idose or HNE by components of methanolic extract fractionation of Zolfino bean, tomato and black cabbage was measured and the results are reported in Fig. 2, Panels $A, B$ and $C$, respectively.

As expected all three species displayed a significant ability in inhibiting AR, however, a different ability to exert differential inhibitory action was observed. In fact, while fractionation of the tomato and the Zolfino bean extracts revealed a number of components able to exert differential inhibition on L-idose reduction with respect to HNE with a different effectiveness and statistical significance, no evidence of differential inhibition appeared among the fractions of the cabbage extracts. The 
Zolfino bean, previously reported as a notable source of ARIs [26] showed a maximal differential inhibition of approximately $30 \%$ ( $<<0.0001)$, (Fig 1. Panel A lower part, fraction 17).

While the significant consistency of this value was verified for different chromatographic runs of different independent bean extracts, some variability was observed for the tomato analysis. In this case although the inhibitory pattern was essentially similar to that reported in Fig. 2, Panel B, an over imposition of the specific peaks was difficult. This is likely due to the profile complexity in the fraction range in which differential inhibition is observed (fractions 11-16, Fig. 2, Panel B,). Thus the number of not well resolved components all able to inhibit the enzyme, may lead to an underestimation of ARDI efficiency.

Finally, it is worth noting that for both Zolfino bean and cabbage (Fig. 2, Panel A, fraction 11 and Panel C, fraction 9, respectively), a differential inhibition with a preferential targeting of $\mathrm{HNE}$ reduction with respect to aldose reduction was observed. Despite the modest statistical significance $(\mathrm{p}<0.05)$, this differential effect accounted for approximately $35 \%$ and $25 \%$, for cabbage and Zolfino, respectively. Although possibly not matching the favorable effects for ARDIs that were being searched for, this evidence, as previously reported for synthetic inhibitors [14], supports the potential of AR to be differentially inhibited.

To conclude, from this preliminary attempt to search for differential inhibitors in edible plants, we believe that there is potentially a new field of investigation. Besides providing a new means of controlling aldose reductase activity, this approach may also be used to reveal how certain foods, highly valuable due to their potentially useful nutraceutical character, may favorably intervene in pathology states linked to the hyperactivity of AR towards specific substrates.

\section{REFERENCES}

[1] J.H. Kinoshita, Cataracts in Galactosemia, Invest. Ophthal. Vis. Sci. 4 (1965) 786-799.

[2] A.Y. Lee, S.S. Chung, Contributions of polyol pathway to oxidative stress in diabetic cataract, FASEB J. 13 (1999) 23-30.

[3] O. El-Kabbani, F. Ruiz, C. Darmanin, R.P. Chung, Aldose reductase structures: implications for mechanism and inhibition, Cell. Mol. Life Sci. 61 (2004) 750-762. doi:10.1007/s00018-003-3403-2. [4] Y. Hamada, N. Araki, N. Koh, J. Nakamura, S. Horiuchi, N. Hotta, Rapid formation of advanced glycation end products by intermediate metabolites of glycolytic pathway and polyol pathway, Biochem. Biophys. Res. Commun. 228 (1996) 539-543. doi:10.1006/bbrc.1996.1695. 
[5] V.J. Stevens, H. Vlassara, A. Abati, A. Cerami, Nonenzymatic glycosylation of hemoglobin, J. Biol. Chem. 252 (1977) 2998-3002.

[6] A. Del Corso, M. Cappiello, U. Mura, From a dull enzyme to something else: facts and perspectives regarding aldose reductase, Curr. Med. Chem. 15 (2008) 1452-1461.

[7] H.M. Krans, Recent clinical experience with aldose reductase inhibitors, Diabet. Med. 10 (1993) S44-S48. doi:10.1111/j.1464-5491.1993.tb00198.x.

[8] M. Foppiano, G. Lombardo, Worldwide pharmacovigilance systems and tolrestat withdrawal, Lancet 349 (1997) 399-400. doi:10.1016/S0140-6736(97)80018-9.

[9] D.L. Vander Jagt, N.S. Kolb, T.J. Vander Jagt, J. Chino, F.J. Martinez, L.A. Hunsaker, R.E. Royer, Substrate specificity of human aldose reductase: identification of 4-hydroxynonenal as an endogenous substrate. Biochim. Biophys. Acta 1249 (1995) 117-126.

[10] S. Srivastava, , A. Chandra, , , A. Bhatnagar, S.K. Srivastava, , N.H. Ansari, Lipid peroxidation product, 4-hydroxynonenal and its conjugate with GSH are excellent substrates of bovine lens aldose reductase. Biochem. Biophys. Res. Commun. 217(1995) 741-746. doi:10.1006/bbrc. 1995.2835.

[11] H. Esterbauer, R.J. Schaur, H. Zollner, Chemistry and biochemistry of 4-hydroxynonenal, malonaldehyde and related aldehydes. Free Radic. Biol. Med. 11 (1991) 81-128. doi:10.1016/08915849(91)90192-6.

[12]. K.V. Ramana, A.A. Fadl, R. Tammali, A.B. Reddy, A.K. Chopra, S.K. Srivastava, Aldose reductase mediates the lipopolysaccharide-induced release of inflammatory mediators in RAW264.7 murine macrophages, J. Biol. Chem. 281 (2006) 33019-33029.

[13] K.V. Ramana, S.K. Srivastava, Aldose reductase: a novel therapeutic target for inflammatory pathologies, Int. J. Biochem. Cell. Biol. 42 (2010) 17-20. doi:10.1016/j.biocel.2009.09.009. [14] A. Del-Corso, F. Balestri, E. Di Bugno, R. Moschini, M. Cappiello, S. Sartini, C. La Motta, F. Da Settimo, U. Mura, A new approach to control the enigmatic activity of aldose reductase, PLoS One 8 (2013): e74076. doi:10.1371/journal.pone.0074076.

[15] M. Cappiello, R. Moschini, F. Balestri, U. Mura, A. Del-Corso, Basic models for differential inhibition of enzymes. Biochem. Biophys. Res. Commun. 445 (2014) 556-560.

[16] R. Moschini, E. Peroni, R. Rotondo, G. Renzone, D. Melck, M. Cappiello, M. Srebot, E. Napolitano, A. Motta, A. Scaloni, U. Mura, A. Del-Corso, NADP ${ }^{+}$-dependent dehydrogenase activity of carbonyl reductase on glutathionylhydroxynonanal as a new pathway for hydroxynonenal detoxification, Free Radic. Biol. Med. 83 (2015) 66-76. doi: 10.1016/j.freeradbiomed.2015.02.001. 
[17] R. Maccari, R. Ciurleo, M. Giglio, M. Cappiello, R. Moschini, A. Del Corso, U. Mura, R. Ottanà, Identification of new non-carboxylic acid containing inhibitors of aldose reductase, Bioorg. Med. Chem. 18 (2010) 4049-4055. doi: 10.1016/j.bmc.2010.04.016.

[18] F. Balestri, M. Cappiello, R. Moschini, R. Rotondo, I. Buggiani, P. Pelosi, U. Mura, A. Del Corso, L-Idose: an attractive substrate alternative to D-glucose for measuring aldose reductase activity, Biochem. Biophys. Res. Commun. 456 (2015) 891_895.

[19] U.K. Laemmli, Cleavage of structural proteins during the assembly of the head of bacteriophage T4, Nature 227 (1970) 680-685.

[20] W. Wray, T. Boulikas, V.P. Wray, R. Hancock, Silver staining of proteins in polyacrylamide gels, Anal. Biochem. 118 (1981)197-203.

[21] M.M. Bradford, A rapid and sensitive method for the quantitation of microgram quantities of protein utilizing the principle of protein-dye binding, Anal. Biochem. 72 (1976) 248-254.

[22] S. Srivastava, S.J. Watowich, J.M. Petrash, S.K. Srivastava, A. Bhatnagar, Structural and kinetic determinants of aldehyde reduction by aldose reductase, Biochemistry 38 (1999) 42-54.

[23] M. Cappiello, M. Voltarelli, M. Giannessi, I. Cecconi, G. Camici, G. Manao, A. Del Corso, U. Mura, Glutathione dependent modification of bovine lens aldose reductase, Exp. Eye Res. 58 (1994) 491-501.

[24] H. Obazatva, L.O. Merola, J. H. Kinoshita, The effects of xylose on the isolated lens, Invest. Ophthalmol. 13 (1974) 204-209.

[25] F. Balestri, M. Cappiello, R. Moschini, R. Rotondo, M. Abate, A. Del Corso, U. Mura, Modulation of aldose reductase activity by aldose hemiacetals, Biochim. Biophys. Acta. 1850 (2015) 2329-2339. doi: 10.1016/j.bbagen.2015.07.007.

[26] F. Balestri, R. Rotondo, R. Moschini, M. Pellegrino, M. Cappiello, V. Barracco, L. Misuri, C. Sorce, A. Andreucci, A. Del Corso, U. Mura, Zolfino landrace (Phaseolus vulgaris L.) from Pratomagno: general and specific features of a functional food, Food Nutr. Res. (2016) http://dx.doi.org/10.3402/fnr.v60.31792. 


\section{Figure legends}

Figure 1 Direct comparison between D- and L-glucose and D- and L-idose as substrates of hAR. Panel A. Reaction rates as a function of the concentration of either D-glucose $(\mathbf{O})$ or L-glucose (O), used as substrates for hAR in the presence of $20 \mathrm{mU}$ of enzyme in the assay mixture. Panel B. Reaction rates as a function of the concentration of either D-idose $(\triangle)$ or L-idose $(\boldsymbol{\Delta})$, used as substrates for hAR in the presence of $8 \mathrm{mU}$ of enzyme in the assay mixture. Error bars (when not visible are within the symbol size) represent the standard deviations of the mean from at least three independent measurements.

Figure 2 Vegetable extract fractionation and AR differential inhibition Each panel refers to the HPLC chromatographic separation profiles (upper part) and to the percentage of inhibition exerted by individual fractions (lower part) on the L-idose (black histograms) and HNE (dashed histograms) reduction. Panels $A, B$ and $C$, refer to the Zolfino bean, tomato and cabbage fractionation, respectively. Eight $\mathrm{mU}$ of hAR were used in the assay. The amount of material used to evaluate the inhibitory ability of each fraction was $1.1,8.5$ and $0.75 \mathrm{mg}$ equivalent of the starting vegetable for Zolfino bean, tomato and cabbage, respectively. Error bars (when not visible are within the symbol size) represent the standard deviations of the mean from at least three independent measurements. The statistical significance of \% differential inhibition on Lidose reduction with respect to HNE reduction is reported as: $* * * * \mathrm{p}<0.0001$; *** $\mathrm{p}<0.0002$, ** $\mathrm{p}<0.001$ and $* \mathrm{p}<0.05$. 
Tab.1 - Relative catalytic efficiency of AR in reducing different classes of substrates

\begin{tabular}{|l|c|c|l|c|c|}
\hline Hydrophobic aldehydes & $\begin{array}{c}k_{s}{ }^{\mathrm{a}} \\
\mathrm{min}^{-1} \mathrm{mM}^{-1}\end{array}$ & Relative $k_{s}{ }^{\mathrm{d}}$ & \multicolumn{1}{|c|}{ Aldoses } & $\begin{array}{c}k_{s} \\
\mathrm{~min}^{-1} \mathrm{mM}^{-1}\end{array}$ & $\begin{array}{c}\text { Relative } k_{s}^{\mathrm{e}} \\
k_{s(\mathrm{~L}) /} k_{s(\mathrm{D})}\end{array}$ \\
\hline propanal & 5.5 & 1.0 & D-glyceraldehyde & $1,860^{\mathrm{b}}$ & \\
\hline butanal & 439 & 79.8 & L-glyceraldehyde & $6,840^{\mathrm{b}}$ & 3.68 \\
\hline hexanal & 3,657 & 664.9 & D-threose & $114^{\mathrm{b}}$ & \\
\hline nonanal & 1,157 & 210.4 & L-threose & $276^{\mathrm{b}}$ & 2.42 \\
\hline trans-2-pentenal & 61.7 & 11.2 & D-arabinose & $0.24^{\mathrm{b}}$ & \\
\hline trans-2-nonenal & 2,058 & 374.2 & L-arabinose & $10.8^{\mathrm{b}}$ & 49.44 \\
\hline 4-hydroxy trans-2-pentenal & 199 & 36.2 & D-xylose & $8.4^{\mathrm{b}}$ & \\
\hline 4-hydroxy trans-2-nonenal & 921 & 167.4 & L-xylose & $0.48^{\mathrm{b}}$ & 0.057 \\
\hline trans,trans-2,4-hesadienal & 139 & 25.3 & D-idose & $4.20^{\mathrm{c}}$ & \\
\hline trans,trans-2,4-nonadienal & 561 & 102.0 & L-idose & $35.75^{\mathrm{c}}$ & 8.51 \\
\hline trans-4-decenal & 1,108 & 201.4 & D-glucose & $0.61^{\mathrm{c}}$ & \\
\hline cis-4-decenal & 862 & 156.7 & L-glucose & n.d & n.d. \\
\hline
\end{tabular}

${ }^{\mathrm{a} D a t a}$ refer to [22]; ${ }^{\mathrm{b}}$ Data refer to [23]; ${ }^{\mathrm{c}}$ Data refer to [18]; ${ }^{\mathrm{d}}$ Relative $k_{s}$, refers to the ratio between the specificity constant of the indicated substrate and propanal; ${ }^{\text {eRelative }} k_{s}$, refers to the ratio between the specificity constant of the $\mathrm{L}$ and $\mathrm{D}$ enantiomers of each aldose; $n$.d. stands for undetectable. 

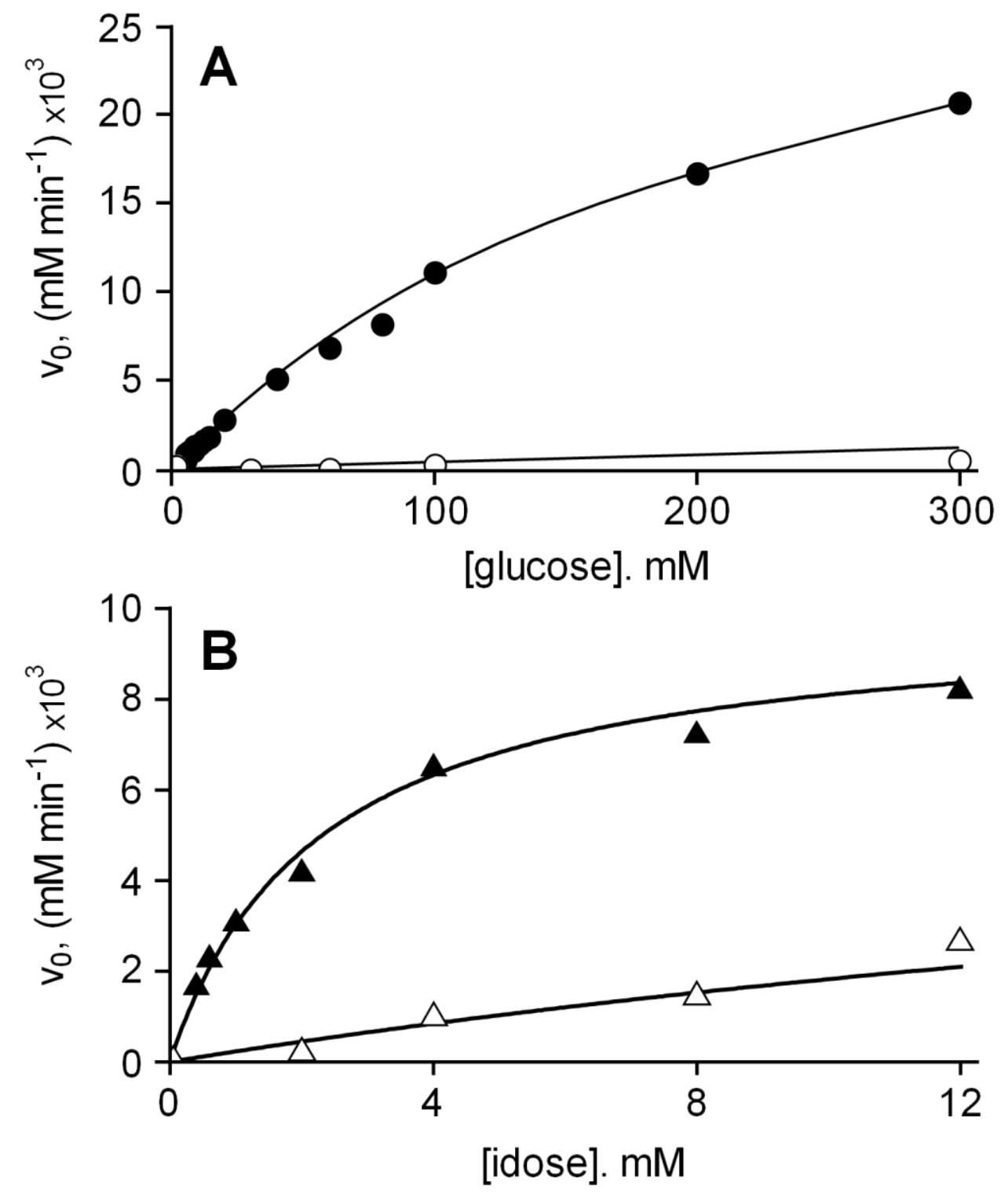

Figure 1 


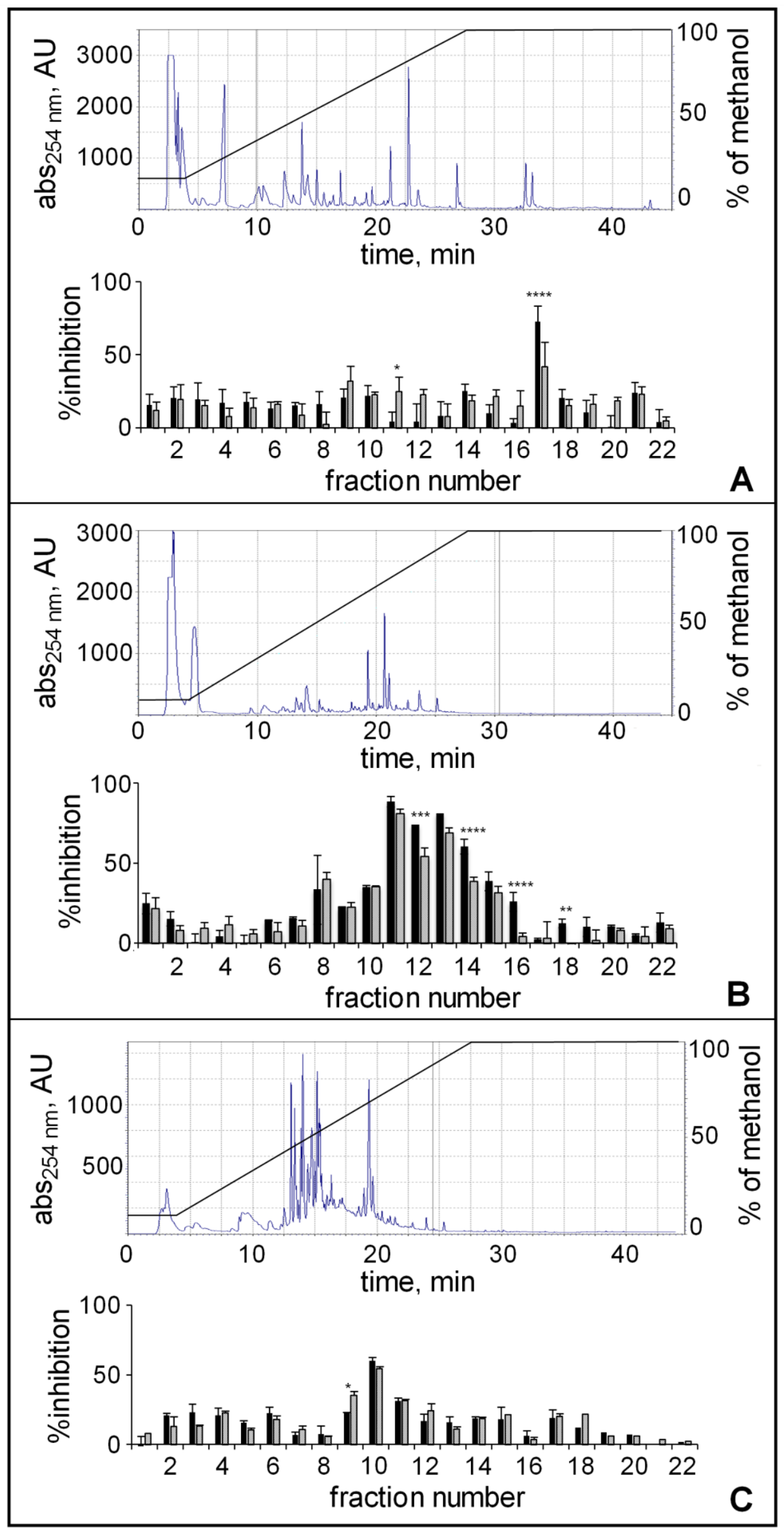

Figure 2 